\title{
False recognition and word length: A reanalysis of Roediger, Watson, McDermott, and Gallo (2001) and some new data
}

\author{
STEPHEN MADIGAN and JAMES NEUSE \\ University of Southern California, Los Angeles, California
}

\begin{abstract}
Roediger, Watson, McDermott, and Gallo (2001) reported a multiple regression analysis of the variables that predicted rates of false recall and recognition across lists in the Deese/RoedigerMcDermott paradigm. They concluded that false recollection was predictable from the backward associative strength of critical words and list words and the veridical recall level of list words, with no independent contribution of frequency, concreteness, or length of critical words. A reanalysis of their data shows that critical word length does contribute to false recognition when it is measured relative to the length of other words in the list. Relative word length in the form of an unsigned $z$-score has a larger correlation with false recognition than any of the variables used by Roediger et al. and is also independently predictive of false recognition. This relationship was confirmed by the results of two recognition experiments in which false positives were significantly less frequent for words of lengths that never occurred in a studied list than for words of lengths that did occur in the study list.
\end{abstract}

The Deese/Roediger-McDermott paradigm for the study of memory errors in recall and recognition consists of the presentation of a list of words (such as light shade table bulb) that are free associates to a critical word (CW; lamp), the $\mathrm{CW}$ itself not occurring in the list. In Roediger and McDermott's (1995) revival of Deese's (1959) experiments and in many subsequent studies, recall intrusions of CWs and false positive rates to CWs as recognition lures have occurred at relatively high rates under a variety of experimental conditions.

Roediger, Watson, McDermott, and Gallo (2001) recently reported an analysis that dealt with the variability in the rate of false memory across different lists and different critical words, observing that "one remarkable outcome that we have noticed while working with these lists is the striking variability they exhibit in eliciting false recall and false recognition of the associatively related critical item" (p. 386). Roediger et al. explored the reasons for this variation, using multiple regression analyses of a set of 55 lists for which information was available about CW and list word characteristics and the false recall and false recognition rates for each list. They analyzed two main types of variables: characteristics of CWs and characteristics of lists. $\mathrm{CW}$ variables were word length, frequency, and concreteness. List variables were measures of associative strength, including forward associative strength (the probability that a given $\mathrm{CW}$ elicited a list word), backward as-

We thank Doug Nelson, Henry L. Roediger, III, Jason Watson, and an anonymous reviewer for helpful comments on the data and analyses. Correspondence concerning this article should be addressed to S. Madigan, Department of Psychology, University of Southern California, Los Angeles, CA, 90089 (e-mail: smadigan@usc.edu). sociative strength (BAS, the probability that a list word elicited the $\mathrm{CW}$ as the first free associate), interitem associative strength (connectivity, based on the number of associative connections among list words), and veridical recall (average recall probability of list words).

The multiple regression analyses for $\mathrm{CW}$ recall and $\mathrm{CW}$ recognition produced essentially the same results: Only BAS and veridical recall contributed significantly and independently to the prediction of false recall and false recognition rates. In particular, $\mathrm{CW}$ length was significantly negatively correlated with recall $(r=-.37)$ and with recognition $(r=-.27)$, but it did not contribute to the multiple correlation in either case. No other variables were significantly correlated with either false recognition or false recall.

Here, we report that the relative length of CWs is more strongly related to false recollection than Roediger et al. (2001) reported for raw word length and that it does contribute to false recognition independently of BAS and veridical recall levels. By relative length, we mean the length of a $\mathrm{CW}$ in comparison with the length of the noncritical (to-be-remembered) words in a particular list. We will first describe the data that suggested the importance of this variable, then will present a reanalysis of Roediger et al.'s data set, and finally will report the results of two recognition experiments that show that the false positive rates to new words are sharply reduced when the length of these words is outside the range of studied word lengths.

\section{Reanalysis of Roediger et al. (2001)}

The analyses reported here were initially suggested by the results of an unpublished study of false memory in free recall (Neuse \& Madigan, 1997). This experiment used 24 


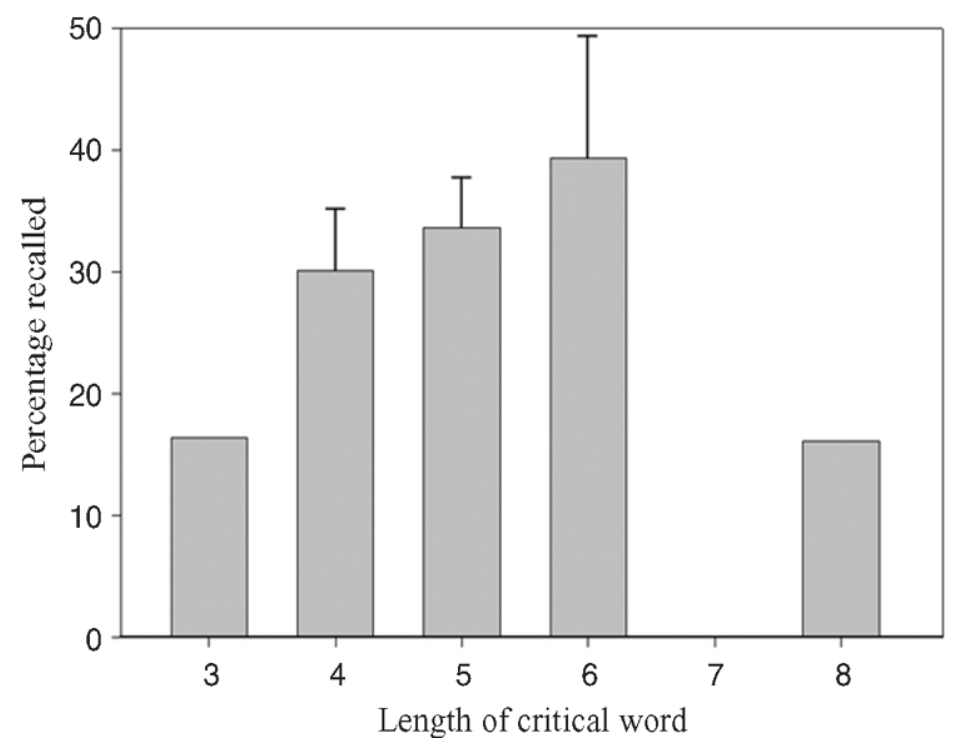

Figure 1. False recall rate as a function of length of critical words. Data from Neuse and Madigan (1997).

of the lists described by Roediger and McDermott (1995). Subjects $(n=130)$ were tested with random samples of 10 of these lists. Presentation was visual at a $1-$ word/sec rate, with written (typed) recall. Critical words were recalled for about $30 \%$ of the subject lists. Figure 1 shows critical word recall as a function of the length of the CWs. These data suggest a curvilinear relationship between $\mathrm{CW}$ recall and CW length, with very short and very long CWs being infrequently recalled. There was only one instance of a three-letter CW (man) and one instance of an eight-letter $\mathrm{CW}$ (mountain) in the set of 24 lists, but the result suggests that a linear correlation analysis of such data could seriously underestimate the strength of the relationship between CW length and false memory.

Prompted by these results, we reanalyzed the full Roediger et al. (2001) data set with the addition of a measure of relative $\mathrm{CW}$ length. For each of the 55 lists used by Roediger et al., we computed the mean and standard deviation of the length of the 15 list words and then expressed CW length as an unsigned standard $(z)$ score. Figure 2 shows the effect of this transformation on the CW length-falserecognition relationship. The top panel shows the scatter plot from Roediger et al.'s data set, with false recognition rate plotted against $\mathrm{CW}$ length. The bottom panel shows false recognition plotted against the unsigned $z$ score as a measure of relative $\mathrm{CW}$ length. The correlation of relative $\mathrm{CW}$ length and false recognition $(r=-.53)$ was noticeably larger than the correlation of raw $\mathrm{CW}$ length and false recognition rate $(r=-.27)$.

We also examined the correlation of several other transformations of raw word length with false recollection. These transformations and their correlations with false recognition were CW length as a deviation score $(r=$ $-.32)$, as a signed $z$-score $(r=-.39)$, and as an absolute deviation score $(r=-.44)$. Not surprisingly, the trans- formations that remove the kind of curvilinearity shown in Figure 1 produce larger correlations with false recollection. The fact that this correlation is largest for the standard score transformations (signed and unsigned) suggests that information about the variability of word length within a list also contributes to the correlation with false recollection. The correlation of raw length and transformed length was also the smallest $(r=.36)$ for the unsigned $z$-score transformation. ${ }^{1}$

In a multiple regression analysis, we substituted the unsigned $z$-score for raw CW length and repeated the analysis reported by Roediger et al. (2001) with the other six predictors they employed. The results appear in Table 1. The major outcome is that relative $\mathrm{CW}$ length contributed to the regression independently of BAS and veridical recall (semi-partial $r=-.23$ ), with a squared multiple correlation of .52, a small increase over that reported by Roediger et al. $\left(R^{2}=.48\right)$. The weights for the other variables shown in Table 1 are quite similar to those reported by Roediger et al. In further analyses, we found that raw CW length never contributed significantly to the regression when relative $\mathrm{CW}$ length was also in the possible set of predictors, irrespective of the order of predictor entry and the variable selection method used. ${ }^{2}$

However, the results of a similar analysis for false recall were different in two important respects. The correlation of relative $\mathrm{CW}$ length and false recall $(r=-.43)$ was only slightly larger than the correlation of raw $\mathrm{CW}$ length and false recall $(r=-.37)$, and in a multiple regression analysis it contributed only marginally as a predictor of false recall, with a semipartial correlation of $-.125(p<.13)$. These results were replicated almost exactly when we analyzed Deese's (1959) free recall data for 23 critical words used by Deese that were also part of Roediger et al.'s (2001) data set. The correlation of relative CW length and 

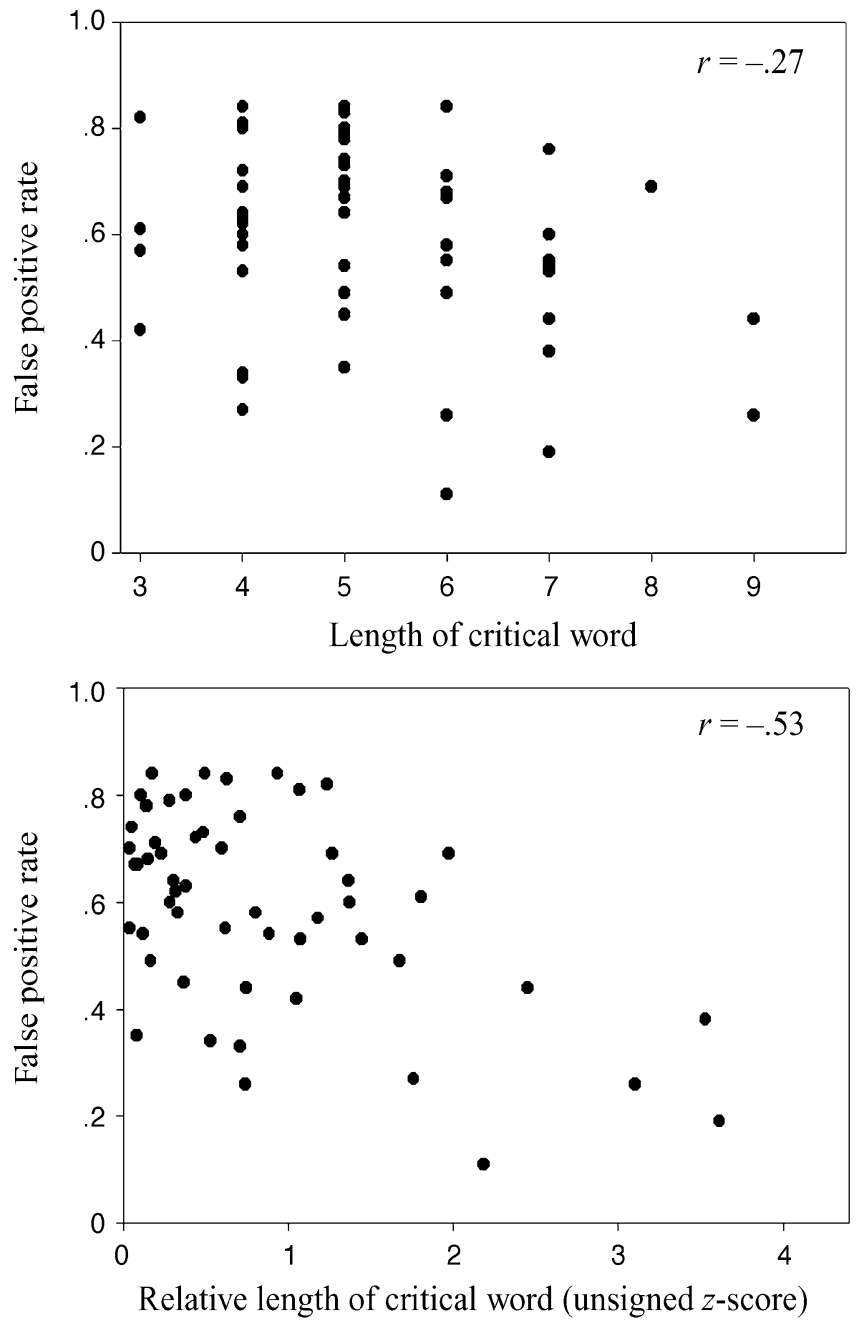

Figure 2. Top panel: length of critical words and false recognition for data from Roediger, Watson, McDermott, and Gallo (2001). Bottom panel: the same data with critical word length as an unsigned $z$-score.

false recall rate was substantial at $r=-.55$, but relative CW length was again only marginally significant in a regression analysis along with the six predictors used by Roediger et al. (2001).

We next will report the results of two experimental tests of the hypothesis that word length distinctiveness can function to reduce false recognition. Suppose that a new word occurs in the test phase of a recognition experiment and that this new word is either shorter or longer than any of the studied list words. Would false positives be less frequent for such words than for new words of lengths that did occur in the study list? This possibility may have already been investigated more than once as a thought experiment, possibly in connection with explaining Deese's (1959) original results with the famous butterfly list, in which this nine-letter $\mathrm{CW}$ was never falsely recalled. Some other support for the hypothesis exists in the results of studies of release from proactive interference, in which changes in word length (Lachar \& Goggin, 1969) or num- ber of syllables (Wickens, 1972) improved performance on the recall trial following the shift.

\section{EXPERIMENT 1}

The first experiment was essentially an attempt to replicate the effect shown in Figure 1, but in a recognition, rather than a recall, experiment and using unrelated words. Subjects saw a list of words of four, five, and six letters and then took a recognition test in which some of the new test words were three and eight letters in length.

\section{Method}

Subjects. Sixty undergraduate students (26 males and 34 females) participated for course credit in the experiment. Data for 2 subjects were discarded due to apparent failures to understand the instructions for recognition testing.

Design and Materials. The experiment was based on a withinsubjects comparison of recognition hits and false positives to study and test words of four, five, and six letters and false positive rates to 
Table 1

Correlations and Multiple Regression Analyses of Roediger,

Watson, McDermott, and Gallo's (2001) Data Set With Relative Critical Word (CW) Length (Unsigned $\boldsymbol{z}$-Score) as a Predictor

\begin{tabular}{|c|c|c|c|c|c|c|}
\hline \multirow[b]{2}{*}{ Variable } & \multicolumn{3}{|c|}{ False Recognition } & \multicolumn{3}{|c|}{ False Recall } \\
\hline & $r$ & $\beta$ & $t$ Value & $r$ & $\beta$ & $t$ Value \\
\hline Log frequency & .21 & +.06 & 0.53 & .16 & -.05 & 0.52 \\
\hline Concreteness & -.13 & -.04 & 0.35 & .11 & +.06 & 0.63 \\
\hline Forward strength & .12 & +.10 & 1.04 & .08 & +.09 & 1.10 \\
\hline Backward strength & .42 & +.27 & $2.31 *$ & .73 & +.65 & $6.71^{*}$ \\
\hline Connectivity & .03 & +.02 & 1.04 & -.04 & -.05 & 0.49 \\
\hline Veridical recall & -.51 & -.44 & $3.83 *$ & -.43 & -.34 & $3.65^{*}$ \\
\hline Relative CW length & -.53 & -.27 & $2.31 *$ & -.43 & -.15 & 1.55 \\
\hline
\end{tabular}

words of three and eight letters. ${ }^{3}$ Words were selected from the MRC Psycholinguistic Database (Coltheart, 1981) to produce sets of words of three, four, five, six, seven, and eight letters. There were 32 words of each of the six word lengths, and these sets were approximately equated for Kučera-Francis written frequency and for imagery ratings, the mean frequency of these sets varying from 44 (three-letter words) to 37 (eight-letter words), with standard deviations ranging from 8.6 (seven-letter words) to 13.3 (eight-letter words). The mean imagery ratings varied from 5.41 (eight-letter words) to 5.66 (fourletter words), with standard deviations ranged from 0.47 (threeletter words) to 0.62 (seven-letter words). These materials were used to create a 60 -item study list and a 76-item test list. The study list consisted of 20 words of each studied word length, presented in a random order. The 76-item test list consisted of a random sample of 12 of the old words for each of these lengths (36 test events), 8 new words for each of these lengths (24 test events), and 8 new words of the two word lengths (three and eight letters) that did not occur in the study list (16 test events). These words were randomly assigned to test positions. Sixty such sets of materials were randomly generated.

Procedure. The testing sessions were conducted by a microcomputer. The subjects first read a set of instructions that directed them to study each list word carefully in anticipation of a later memory test. They were instructed at this point only that they would not have to remember the order of occurrence of words. The subjects then saw the study list one word at a time at a rate of 1 word/sec, presented in the center of an otherwise blank display. Test instructions occurred immediately after list presentation. These instructions were for a standard old-new recognition procedure, directing the subjects to respond old if the displayed test word had appeared in the study list and otherwise, to respond new. The subjects responded in test trials by a mouse click on buttons labeled old and new that appeared below the test word display. This test was self-paced.

\section{Results}

For all statistical tests, the rejection level was set at .05. Figure 3 summarizes the results of the experiment in the form of hit and false positive rates for old words of four, five, and six letters and false positive rates for new threeand eight-letter words (the word lengths that did not occur in the study list). The proportion of false positive rates was much lower (.134) for three- and eight-letter words, as compared with the mean $(0.271 \%)$ for new words of studied lengths $\left[F(1,56)=40.80, M S_{\mathrm{e}}=.029\right]$. For words of four, five, and six letters, there was no reliable variation in

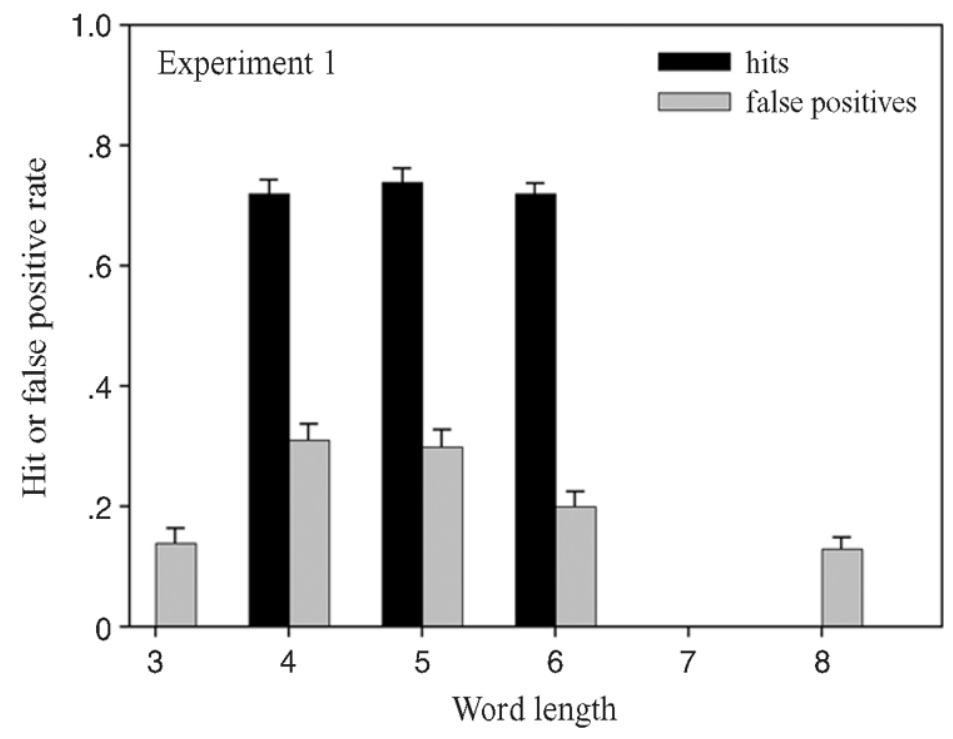

Figure 3. Hit and false positive rates in Experiment 1with three- and eightletter words occurring only as new items in the recognition test. 
hit rates as a function of word length $[F(2,114)=1.04$, $\left.M S_{\mathrm{e}}=0.012\right]$, but there was significant variation in the false positive rates $\left[F(2,114)=10.19, M S_{\mathrm{e}}=0.019\right]$, with significantly fewer false positives for six-letter words. Recognition accuracy (hits plus correct rejections) also increased significantly with word length $[F(2,114)=8.17$, $\left.M S_{\mathrm{e}}=0.069\right]$.

The apparent reduction in the rates of false positives for words of lengths that did not occur in the study list replicates and extends findings reported by Higham and Brooks (1997) that we were unaware of while conducting Experiment 1 . They showed that following a study trial of a list of 947 - and 8-letter words, the false positive rates for new test words of 5, 6, 9, and 10 letters were significantly lower than those for new test words of 7 and 8 letters.

\section{EXPERIMENT 2}

The second experiment had two purposes. One was to investigate the boundary conditions of the nonstudied word length effect: Would false positive responses also be less frequent for nonstudied word lengths when these word lengths were embedded in the range of studied word lengths, rather than being shorter or longer than the lengths of any studied words (e.g., new words of five or six letters following study of a list of three, four, seven, and eightletter words)?

The second aim of Experiment 2 was to address some problematic aspects of design in Experiment 1, some of which were also present in Higham and Brooks's experiments. Experiment 1 lacked controls for the two nonstudied word lengths (three- and eight-letter words). Figure 1 contains a suggestion that the false positive rate might decline with increasing word length, which complicates the interpretation of the reduced rate of false positives for eight-letter words in Experiment 1. In addition, although Experiment 1 used sets of words of varying length with approximate matching of word frequency and word imagery across word lengths, there may be other word attributes confounded with word length in the relatively small sets of words for each word length. We examined one such variable (rated meaningfulness), based on the Colorado norms (Toglia \& Battig, 1978) that are included in the MRC database (Coltheart, 1981). Rated meaningfulness scores were available for $176(66 \%)$ of the 264 words used in Experiment 1. The mean ratings for words of lengths of three to eight letters were 4.83, 4.56, 4.77, $4.69,4.49$, and 4.66 , respectively. There was marginally significant variation across these means arising from the contrast of word lengths of three to four letters, but these data suggest that meaningfulness cannot account for the effects in Experiment 1, at least with the present sets of words. Of course, there may be additional word characteristics that need to be taken into account. A particularly important one for recognition testing is associative similarity or connectivity. To quote from a reviewer, "the reader needs assurance that the different-length lures did not have weaker relationships to the study words than the same- length lures." Rather than attempt further matching of word characteristics of words of different lengths, we took a more direct approach and simply compared the false positive rates to word lengths that did not occur in a study list with false positive rates from a control study list that did include these word lengths.

\section{Method}

Subjects. The subjects were 192 undergraduates participating for course credit. The data for 12 randomly selected subjects were discarded to produce equal sample sizes for the three treatment conditions.

Design and Materials. The subjects within each testing session group were assigned at random to one of three different kinds of study lists constructed from the word pool used in Experiment 1. In the basic control condition, the subjects studied words of three, four, five, six, seven, and eight letters. In the two experimental conditions, the subjects saw a list that included only four word lengths. For one of these conditions, the study list consisted of words of four, five, six, and seven letters, with a test list that included new words of these lengths in addition to new words of three and eight letters. In the other condition, the four studied word lengths were three, four, seven, and eight letters, with a test list that included new words of these lengths in addition to new words of five and six letters. The study lists for these two conditions consisted of 16 words of the four studied lengths. In order to maintain a constant study list length of 64 words, the control list consisted of sets of 10 words of lengths of three, five, six, and eight letters and two sets of 12 words of four and seven letters.

The test list of 96 words was also the same length for all conditions. In the control condition, there were 8 old and 8 new words for each of the six studied word lengths. In the two experimental conditions, there were 10 old and 10 new words for the four studied lengths and 8 new words for the two nonstudied lengths.

Twelve sets consisting of two experimental and one control list were created by three independent samplings from the six sets of 32 words of each length, crossed with four counterbalanced test orderings of words. The study sequences were randomized with respect to word length and serial position.

Procedure. Testing was conducted in eight groups of from 18 to 32 undergraduate students. Within each group, the subjects were assigned to treatment conditions by random distribution of study-test booklets. Each testing session started with instructions to the subjects to study each presented word carefully in anticipation of a later memory test that would not require remembering the order of the words. The subjects were then given $70 \mathrm{sec}$ to scan the 64 -word study list that was printed in four columns on a single page. Recognition test instructions were given immediately following list presentation and directed the subjects to circle each word on the 96-item test page that they thought had occurred in the study list. The subjects were also instructed that half of the test items had occurred in the study list. The subjects were allowed $5 \mathrm{~min}$ to complete the test.

\section{Results}

Figure 4 shows the proportions of false positives for three- and eight-letter words and five- and six-letter words when these word lengths occurred or did not occur in the study list. The main result is clear and consistent: False positives were uniformly lower for nonstudied than for studied word lengths, in an experimental comparison that controlled word characteristic effects across word lengths. This was the case for the word lengths (three and eight letters) that were outside the range of studied lengths $\left[F(1,118)=9.92, M S_{\mathrm{e}}=0.008\right]$, as well as for word lengths (five and six letters) that were spanned by the range of 


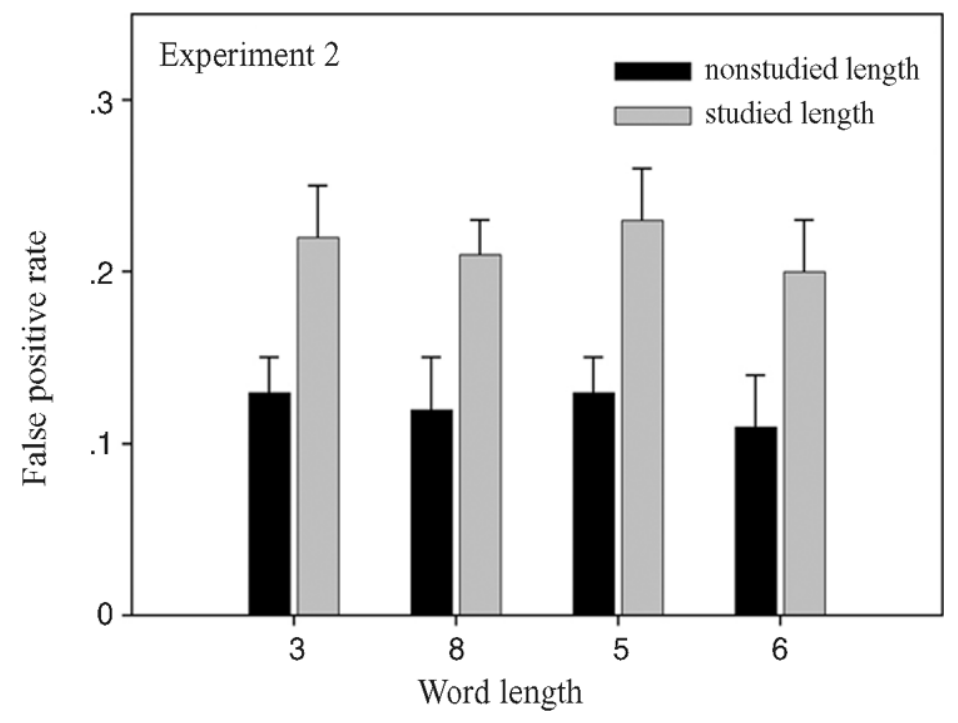

Figure 4. False positive rates for studied and nonstudied word lengths, Experiment 2.

studied word lengths $\left[F(1,118)=6.47, M S_{\mathrm{e}}=0.011\right]$. The average hit rate was .83 and did not vary reliably over word lengths (as in Experiment 1) or conditions.

\section{GENERAL DISCUSSION}

A central finding of Roediger et al. (2001) was that only the list variables of $B A S$ and veridical recall independently contributed to a modeling of false recall and false recognition rates by multiple regression. Item variables $(\mathrm{CW}$ frequency, concreteness, and word length) had no independent contribution. Our analyses of the recognition data indicates that there is, in fact, at least one item variablerelative $\mathrm{CW}$ length - that is also predictive of false recollection. The correlation and regression analyses we have reported used a measure of word length that incorporated information about relatively short CWs and relatively long CWs in a way that the relationship of word length to false recognition rates was linear, with the result that, at least for recognition, CW length was strongly associated with false recollection and was also independently predictive of false recognition.

The two recognition experiments confirm and extend the results and causal implications of the correlational analyses. In Experiment 1, the false positive rate to new test words was halved if the test words were of lengths that did not occur in the study sequence. In Experiment 2, this effect was replicated and also occurred for new test words whose lengths did not occur in the set of studied word lengths but were, instead, spanned by the range of length of studied words.

Roediger et al. (2001, p. 394) offered a straightforward interpretation of the correlation of word length and false recollection that also suggests why relative $\mathrm{CW}$ length was more influential for false recognition than for false recall: "If words in the list are generally shorter [than the CWs], then during retrieval the critical items that are longer... should be more easily discriminated from the list items." On this view, one reason for the differences between the results of the present recall and regression analyses is that word length in a visual recognition task simply provides more data for data-driven recognition process (Jacoby \& Dallas, 1981) than does a recall test. A much earlier statement about the importance of this kind of formal similarity makes the same point:

If we required $S$ to learn a list of adjectives and then placed the adjectives among a group of nonsense syllables, $S$ would probably show very small loss in retention. Obviously, the similarity of the test material to the other material is an important variable which determines the recognition score. (Underwood, 1949, p. 512)

The present results make interesting contact with those of two other kinds of recognition experiments in which the degree of overlap of study item and test item characteristics has been examined. Our main results can be described as examples of the extralist feature effect reported by Mewhort and Jones (2000), in which test probes that did not have any features in common with items in a relatively short (three- or four-item) study list were quickly and confidently rejected. Parkin et al. (2001) used a similar approach to study the effects of the overlap of letters making up studied words and test distractor words. In the overlap condition, list words and distractors were both drawn from the full set of 26 letters of the alphabet. In the no-overlap condition, the letters used for study and distractor words were selected from two disjoint subsets of the alphabet. For present purposes, the key finding of their experiments was that the reduced formal similarity of new test items to studied items in the no-overlap condition produced a large decrease in the false positive rate. 
Two of the conclusions reached by Parkin et al. (2001) seem to apply directly to an understanding of the present findings. The first is that recognition memory "can be driven by relatively low-level (sublexical, perceptual) characteristics of the stimuli" (p. 816); the second is that "perceptual distinctiveness can be protective against false recognition" (p. 817). In general, the present results and those of Higham and Brooks (1997) and Parkin et al. suggest that recognition can be quite sensitive to test events that have characteristics whose values fall outside the range of those characteristics in the set of experienced or studied events, even for the case of a noncontinuous range, as in Experiment 2. Parkin et al. also found that their subjects appeared to be generally unaware of or, at least, unable to articulate the structure of study and test lists, and Higham and Brooks's results indicated that the word length effect occurred independently of subjects' awareness of the "rules" of study and test list composition. Our experiments did not include an assessment of subjects' awareness of word length nonoverlap for new items, but no subject ever spontaneously reported noticing this, so that the word length effect may well be based on implicit processes or, as Higham and Brooks put it, on the tacit sensitivity of subjects to the structure or statistics of experimental materials.

\section{REFERENCES}

Coltheart, M. (1981). The MRC psycholinguistic database. Quarterly Journal of Experimental Psychology, 33A, 497-505.

DEESE, J. (1959). On the prediction of occurrence of particular verbal intrusions in immediate recall. Journal of Experimental Psychology, 58, $17-22$.

HiGHAM, P. A., \& BRooks, L. R. (1997). Learning the experimenter's design: Tacit sensitivity to the structure of memory list. Quarterly Journal of Experimental Psychology, 50A, 199-215.

JACOBY, L. L., \& DALlAS, M. (1981). On the relationship between autobiographical memory and perceptual learning. Journal of Experimental Psychology: General, 110, 306-340.

LACHAR, B., \& GoGGIN, J. (1969). Effects of changes in word length on proactive interference in short-term memory. Psychonomic Science, 17, 213-214.

Mewhort, D. J. K., \& Johns, E. E. (2000). The extralist-feature effect:
Evidence against item matching in short-term recognition memory. Journal of Experimental Psychology: General, 129, 262-284.

NeuSE, J., \& Madigan, S. (1997). False recall rates and item format. Unpublished manuscript, University of Southern California.

Parkin, A. J., Ward, J., Squires, E. J., Furbear, H., Clark, A., \& Townshend, J. (2001). Data-driven recognition memory: A new technique and some data on age differences. Psychonomic Bulletin \& Review, 8, 812-819.

RoEDIGER, H. L., III, \& McDermott, K. B. (1995). Creating false memories: Remembering words not presented in lists. Journal of Experimental Psychology, 21, 803-814.

Roediger, H. L., III, WAtson, J. M., McDermott, K. B., \& Gallo, D. A. (2001). Factors that determine false recall: A multiple regression analysis. Psychonomic Bulletin \& Review, 8, 385-407.

Toglia, M. P., \& BatTig, W. (1978). Handbook of semantic word norms. Hillsdale, NJ: Erlbaum.

Underwood, B. J. (1949). Experimental psychology. New York: Appleton-Century-Crofts.

WICKENS, D. D. (1972). Characteristics of word encoding. In A. W. Melton \& E. Martin (Eds.), Coding processes in human memory (pp. 191215). New York: Wiley.

\section{NOTES}

1. Some additional measures of $\mathrm{CW}$ distinctiveness were examined as well. For example, we simply counted the number of words in each list that were the same length as the $\mathrm{CW}$ for each list, and we also looked at features that contribute to orthographic distinctiveness, such as ascenders and descenders. Some of these variables were significantly correlated with false recognition, but none of them was as strongly correlated with false $\mathrm{CW}$ recognition as was relative length as an unsigned $z$-score, and none of them contributed independently as predictors in regression analyses.

2. Figure 2 might suggest that relative word length correlation with false recognition is based on a relatively small number of words that generate large $z$-score values. Deleting the rightmost six points in Figure 2 does eliminate relative word length as a predictor, and the effect of interest would be more convincing if there were more such items in the data set. In any event, the results of the two experiments that follow up the correlational analyses indicate that the relationship shown in Figure 2 is more than an artifact produced by outliers.

3. We used eight-letter, rather than seven-letter, new words because of some pilot testing that we thought suggested that discriminability declined with increasing word length.

(Manuscript received July 1, 2002; revision accepted for publication July 31, 2003.) 